\title{
Fabrication of a Biofilter Using Anodic Reaction for Filtering Blood
}

\author{
Duk-Soo Eun, Jong-Hyeon Jeong, Jang-Kyoo Shin and Jong-Hyun Lee* \\ Department of Electronics, Graduate School, Kyungpook National University, \\ 1370 Sankyuk-Dong, Buk-Ku, Daegu 702-701, Republic of Korea
}

(Received July 30, 2008; accepted November 25, 2008)

Key words: biofilter, anodic reaction, micropore, PDMS

In this paper, we describe the fabrication of a biofilter using a porous layer formed by an anodic reaction of (110) silicon to increase the width of pores and decrease the width of the walls between pores. The filter was fabricated from micropores formed through post-treatment and membrane structure formation. The fabricated filter, combined with a polydimethylsiloxane (PDMS) module and a filter structure, can be loaded with a lab-ona-chip for filtering blood.

\section{Introduction}

In general, both protein and DNA chips require advanced laboratory processes involving separation and refinement. Lab-on-a-chip technology in which one step is developed to perform the categorization of a specimen into elements has progressed to a point close to the realization of such chips. Therefore, microfluidic technology that can supply materials to microchannels and nano-electro-mechanical systems (NEMS) is required.

A microchannel can be used to conduct experiments and research using very small amounts of samples and specimens in a next-generation diagnosis system. Lab-on-achip technology commonly uses chemical methods to filter specimens. These methods may be effective in separating chemical samples.

When separated by a chemical method, specimens can be denatured by a chemical reaction. To minimize denaturation and separate nondenatured specimens from chemical materials, mechanical filtering may be considered as a viable alternative. For this purpose, in 2004, Grigoras et al.(1) produced a porous silicon membrane. Pores with widths of $1 \mu \mathrm{m}$ or less were formed vertically with pore walls of approximately $1 \mu \mathrm{m}$ thickness in a porous layer that was $40 \mu \mathrm{m}$ thick. This filter was designed to sieve microbeads from a protein chip but has been shown to filter other materials by adjusting the width of the pores. The filter, however, has a relatively thicker wall than the pores, so that the maximum porosity rate is no higher than $43 \%$. The low rate of porosity affects

*Corresponding author: e-mail: jhlee@ee.knu.ac.kr 
microfluid flow. A higher porosity rate can increase the efficiency of filtering so that it has minimal effect on microfluidic flow.

In this paper, we describe the fabrication of a biofilter using a porous layer formed by an anodic reaction of (110) silicon to increase the width of the pores and decrease the width of the walls between pores. The channeling effect in which (110) silicon can be produced structurally may also improve porosity. The filter was made out of the fabricated micropores through posttreatment and membrane structure formation. The fabricated filter, combined with a polydimethylsiloxane (PDMS) module and a filter structure, can be loaded with a lab-on-a-chip for filtering blood.

\section{Anodic Reaction Theory}

Porous silicon was accidentally discovered about 50 years ago while researchers were trying to electropolish silicon. An anodic reaction induced using concentrated hydrofluoric acid (HF) is one of the most widely used methods to create a porous silicon layer (PSL) from bulk silicon. Under certain conditions for electrolysis, the localized dissolution of silicon may occur, which creates pores within bulk silicon. ${ }^{(2-5)}$ This process can achieve volume porosities between 5 and $85 \%$ with pores of 1 to $100 \mathrm{~nm}$ radius. These properties are dependent on silicon type, the type of dopant in the substrate and electrolysis parameters. ${ }^{(6)}$ Any thickness of porous silicon can be obtained; the thickness is determined by the amount of electrical charge that is exchanged during the reaction.

To carry out the anodic reaction, the wafer is placed in HF solution, electrodes are placed on opposite sides of the wafer and then a potential is applied across the two electrodes. This creates a potential field across the wafer and a charge in the solution. The schematic setup for electrochemical silicon etching is shown in Fig. 1.

Beale et al. ${ }^{(7)}$ observed that the resistivities of dry porous silicon and intrinsic Si are very similar. From this fact, it was concluded that the interpore regions have no mobile

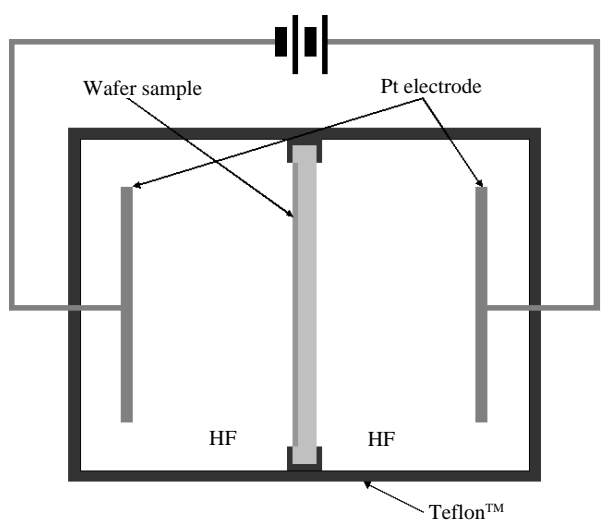

Fig. 1. Schmatic of process for anodic reaction. 
carriers, holes or extra electrons, owing to the overlapping of the depletion regions. As a consequence, the current was directed to pores owing to the high resistance of the depleted porous structure. To achieve the appropriate depletion of the silicon, it is necessary to assume Fermi level pinning at the midband gap. There are two processes by which porous silicon can form during electrolysis, as described in eqs. (1) and (2) below.

$$
\begin{gathered}
\mathrm{Si}+2 \mathrm{HF}+(2-n) h^{+} \rightarrow \mathrm{SiF}_{2}+2 \mathrm{H}^{+}+n e^{2-} \\
\mathrm{SiF}_{2}+2 \mathrm{HF} \rightarrow \mathrm{SiF}_{4}+\mathrm{H}_{2}(n<2) \\
\mathrm{Si}+4 \mathrm{HF}+(4-\lambda) h^{+} \rightarrow \mathrm{SiF}_{4}+4 \mathrm{H}^{+}+\lambda e^{-} \\
\mathrm{SiF}_{4}+2 \mathrm{HF} \rightarrow \mathrm{H}_{2} \mathrm{SiF}_{6}(\lambda<4)
\end{gathered}
$$

The symbols $n$ and $\lambda$ are the reaction coefficients. The method described in eq. (1) depends on the availability of less than 2 holes in each silicon, while the process described in eq. (2) depends on the availability of less than 4 holes.

\section{Experiment and Fabrication}

\subsection{Conditions limiting porous layer formation}

Various external factors affect an anodic reaction, such as voltage, current, environmental temperature, HF solution concentration, and the type and state of samples. Several variables have been used to assess the accuracy of the experiment. Temperature is an important variable in an anodic reaction, regardless of the material used. In other words, temperature has a significant effect on the HF solution reaction as well as on the interface of samples, which changes not only the reaction rate but also the type of material. In the case of silicon, temperature becomes a variable related to the formation of a porous layer and an anodic oxide. This is due to the high potential difference at which the interface produces an anodic oxide rather than a porous layer when the temperature is higher than a specific value.

In this study, the laboratory was maintained at a constant temperature of $26^{\circ} \mathrm{C}$. The use of a low-HF-concentration solution led to ease of channeling. The low HF concentration in the solution did not exceed $20 \%$, and in this study, only $10 \%$ HF solution was used. The ratio of ethanol to water was maintained at 1:5. As the test results show, when the solution contained more ethanol than water, the bubbles on the silicon surface had a less prominent effect on the silicon surface reaction. On the other hand, an increase in the bubble content on the platinum electrode resulted in a higher voltage relative to the current being measured. If ethanol is contained in the solution, bubbles occuring on the silicon surface do not dissipate quickly, and surface uniformity decreases.

In this study, a 4-inch 'p'-type (110) silicon layer having a specific resistance range of 3-9 $\Omega \cdot \mathrm{cm}$ was used. A diced sample is commonly used to conduct experiments. However, in the study, a 4-inch wafer was used because it reduced the adverse effect on the distribution of the electric field. Figures 2(a) and 2(b) show scanning electron microscopy (SEM) images of the anodic reaction to remove thermally grown $\mathrm{SiO}_{2}$ and deposited $\mathrm{Si}_{3} \mathrm{~N}_{4}$. Pores were grown on the sample together with partial channels, the 


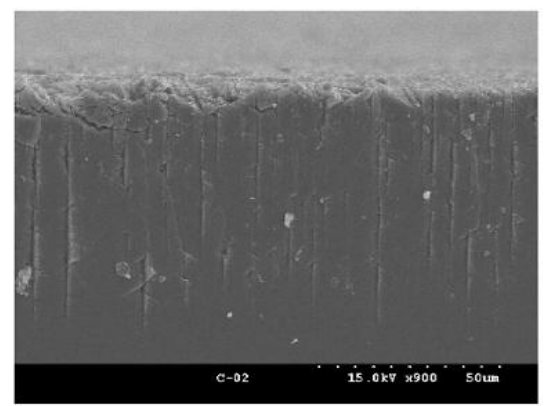

(a)

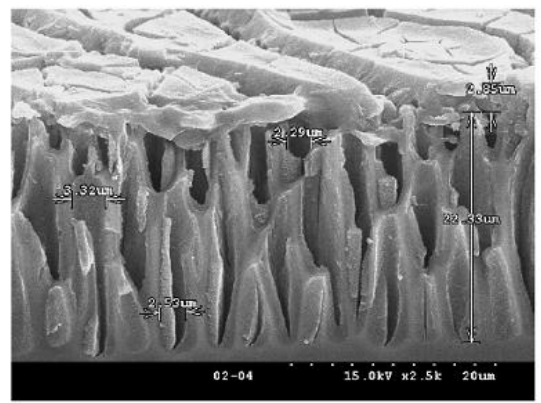

(b)

Fig. 2. Structural distinction of pores in surface film: (a) presurface treatment of thermal $\mathrm{SiO}_{2}$, (b) presurface treatment of deposited $\mathrm{Si}_{3} \mathrm{~N}_{4}$.

number of which depended on crystal direction (Fig. 2(a)). The growth of oxide on the silicon surface produced many defects at the interface between oxide and silicon, so that the electric field applied to the depletion layer containing the defects that produced an anodic reaction had an effect more prominent than the channeling effect. The interfaces of silicon produced no defects because the sample (Fig. 2(b)) was deposited with $\mathrm{Si}_{3} \mathrm{~N}_{4}$. In other words, $\mathrm{Si}_{3} \mathrm{~N}_{4}$ can better protect the silicon surface, and defects affect channeling effects. $^{(7-13)}$

\subsection{Condition of (110) silicon anodic reaction for filter fabrication}

In this study, a filter for filtering red blood cells as well as white blood cells of highviscosity blood was designed. Environmental conditions for the anodic reaction were set considering the pore width and strength of porous layers and the viscosity with regard to filtering the materials. The pore width was based on the size of red blood cells since white blood cells are much larger. Red blood cells have an approximate size of $7 \mu \mathrm{m}$. However, they can penetrate into pores with a width of $5 \mu \mathrm{m}$ owing to the elasticity of the cells. If the width of the pores was too small, the porous layer could have been destroyed owing to the viscosity of the materials. In this study, the pores were designed to have widths of $2-4 \mu \mathrm{m}$.

The depth of the pores was chosen considering the anodic reaction time as well as the porous structure. After the anodic reaction, the sample produced porous layers and we changed the posttreatment process to one based on the thickness of approximately $50 \mu \mathrm{m}$. However, in a porous layer having a thickness of approximately $60 \mu \mathrm{m}$ or more, low-porosity walls were formed between the pores after the posttreatment or the shape of the pores changed. The porous layer should be as thick as possible, considering filter strength, in order to eliminate changes in the structure of the pores, so that the maximum depth of the pores is approximately $40 \mu \mathrm{m}$ after the anodic reaction. Figure 3 shows SEM images of the sample fabricated under the experimental conditions. ${ }^{(7-13)}$ 


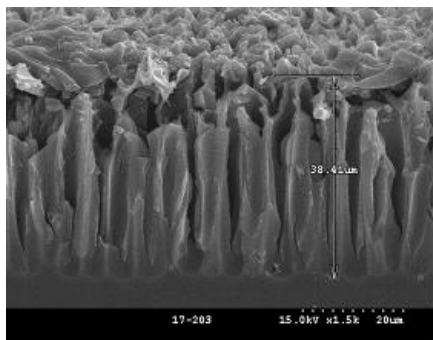

(a)

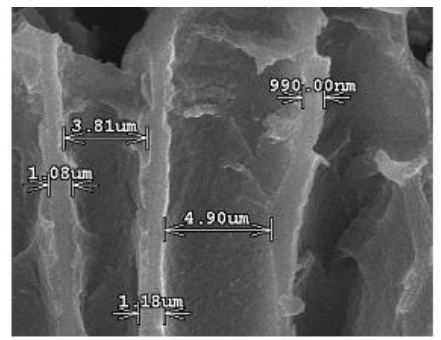

(b)

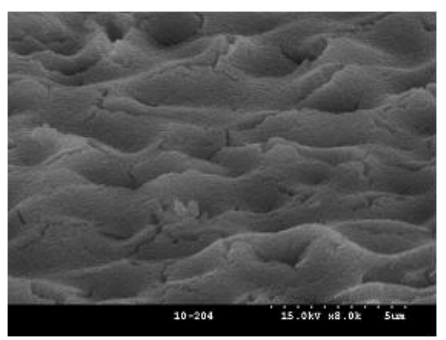

(c)

Fig. 3. SEM images of the sample fabricated under experimental conditions: (a) cross-sectional view, (b) cross-sectional detailed view, and (c) detailed view of surface.

\subsection{Fabrication of a biofilter}

Our fabrication method was different from existing methods and incorporated the membrane production method after the porous layer was produced. In accordance with making the filter porous, the front of the sample makes use of all the membranes as a filter.

Figure 4 shows the schematic of the filter fabrication process. Figures 4(a) and 4(b) show the formation of porous layers through an anodic reaction by depositing aluminum on the rear of the sample. Figure 4(c) shows the use of $\mathrm{NaOH}$ and HF solutions in the posttreatment process to remove minute holes as well as the anodic oxidation film. As shown in Fig. 4(d), the walls of the pores were strengthened to grow a sufficiently thick oxide film to prevent deformation during other processes. As shown in Figs. 4(e)-4(g), the filter is formed by etching after a pattern is made on the back side of a sample.

During the anodic reaction, the (110) silicon sample formed a porous structure divided into top and bottom layers, as shown in Fig. 5. The porous structure can be divided into an anodic oxidation film and a layer with minute pores. The bottom porous layer was filled with minute pores within the macropore surface (Fig. 6(a)). Not only the wall but also the layer of minute pores could be divided by an anodic oxide film. Figure 6(b) shows the border between the layer of minute pores on the wall and the silicon structure after the sample was immersed in $10 \mathrm{wt} \% \mathrm{NaOH}$ solution for approximately $20 \mathrm{~s}$ to remove the layer of minute pores at the bottom and top layers. $\mathrm{NaOH}$ has a very low etching rate for silicon; thus, it easily reacts with the layer of minute pores, owing to the large surface area, and only removes the layer of minute pores without causing damage to the silicon. The surface has a very loose thin structure despite the presence of an anodic oxide film, so that the bottom layer of minute pores could be removed with no effect on the oxide film. Figure 7 shows a cross-sectional SEM image of the sample with the layer of minute pores removed using $\mathrm{NaOH}$ solution. The sample was immersed in $49 \mathrm{wt} \%$ HF for approximately 3 min to completely remove the anodic oxide film on the macropore surface, after removing all the layers of minute pores on the bottom and top macropore layers. 


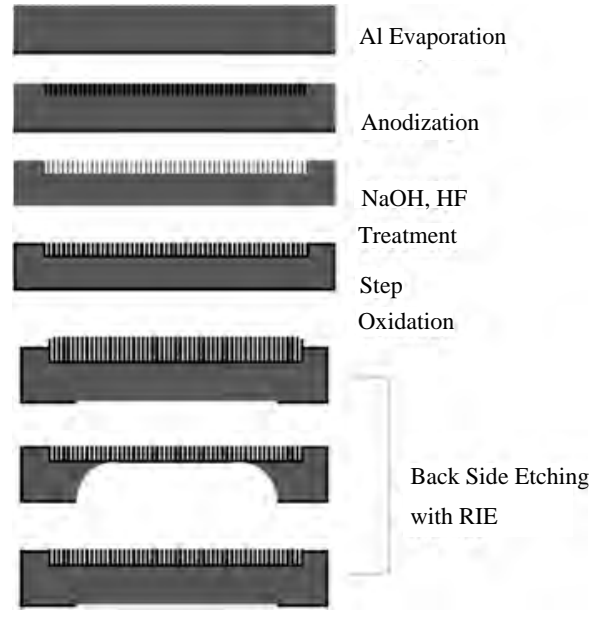

Fig. 4. Schematic of filter fabrication process.

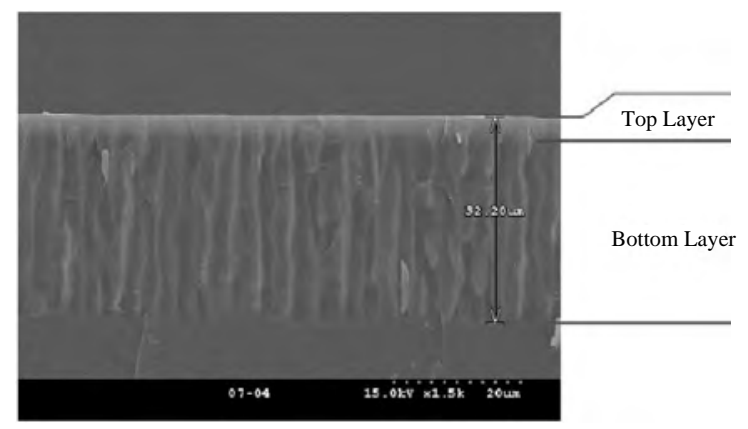

Fig. 5. Porous structure of the fabricated sample.

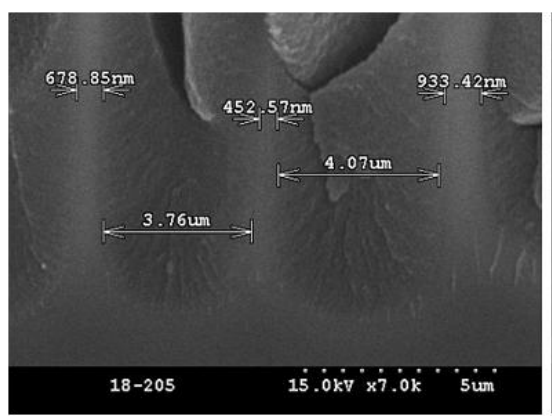

(a)

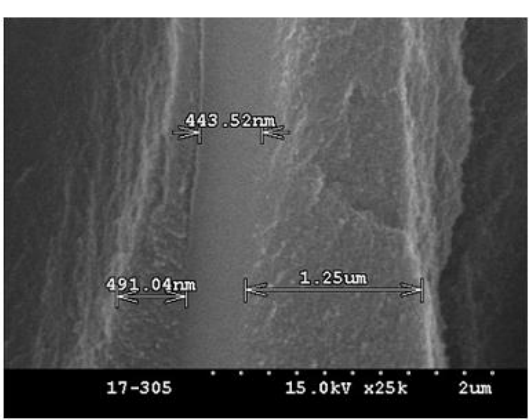

(b)

Fig. 6. Structure of bottom porous layer: (a) bottom of porous layer with anodized oxide and (b) detailed view of wall between pores. 


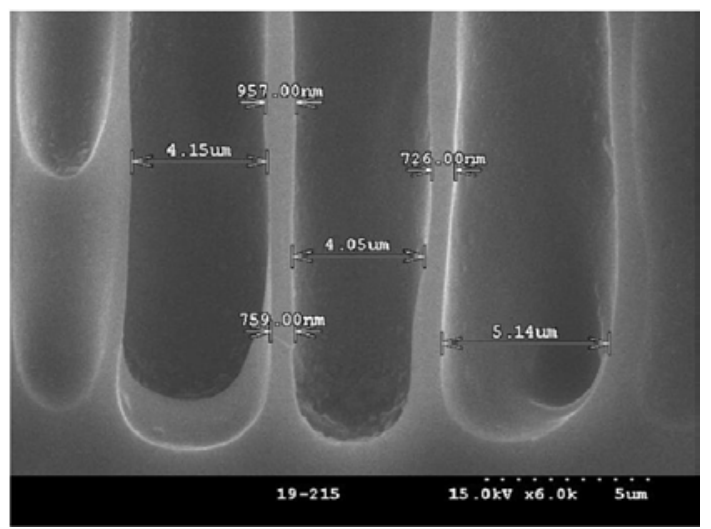

Fig. 7. Porous layer after $\mathrm{NaOH}$ treatment.

Reactive ion etching (RIE) on the rear of the substrate was used to etch silicon to minimize damage to the porous layer. RIE has isotropic and anisotropic features depending on the experimental conditions. The experimental conditions in this study were set to produce an arch-shaped silicon substrate and conduct isotropic etching, as shown in Fig. 8. PVC adhesive sheets were used for etching because the photoresist penetrated into the porous layers. The pattern was $2 \mathrm{~mm}$ in diameter, the same size as the circular silicon tube that was connected to the top layer. $\mathrm{SF}_{6}$ gas was used; Ar active gas was not used, because active gas could have destroyed the oxide film by changing the etching rate over a process lasting several hours. The sample shown in Fig. 9(a) maintained its original pore structure after the RIE process, while that of Fig. 9(b) had a different pore structure resembling a colony of cells. With regard to the sample in Fig. 9(b), the walls between the pores did not change into a perfect oxide film; thus, the wall interior was still composed of silicon. After the completion of the RIE process, the silicon in the wall was etched. It changed into a porous layer having a very high porosity, causing the remainder of the pores to resemble those in Fig. 9(b).

\section{Characteristics}

\subsection{Combination of filter and PDMS}

Figure 10 shows the schematic of the fabricated filter combined with PDMS. In this study, only the filter was produced, and the inlet for supplying the specimen to the filter was made of a silicone hose as well as PDMS. The filter itself was hydrophilic owing to the oxide film on the surface of the silicon, while PDMS was hydrophobic; thus, the connection between the PDMS and the filter was not strong. High-polymer chemicals such as dry film resist (DFR) can perform thermal bonding. However, the filter layer may be exposed to heat stress and have the disadvantage of a narrow seaming area. In this study, glue bonding was used to attain good adhesion at a narrow area without use of heat. Figure 11 shows the fabricated filter used for measurement purposes. 


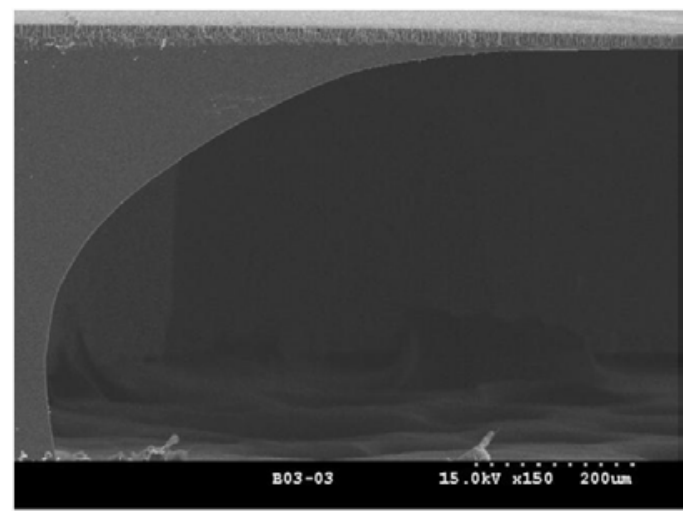

Fig. 8. Cross-sectional SEM image of filter.

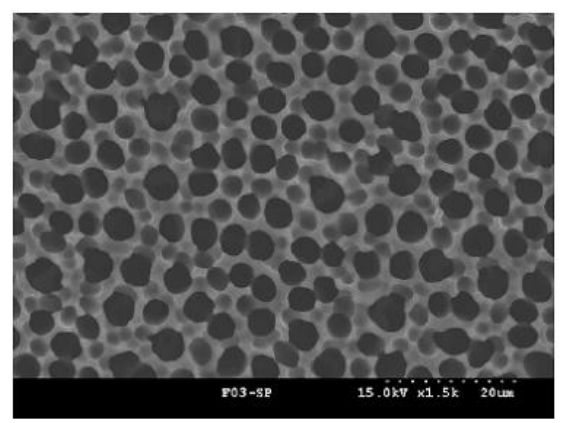

(a)

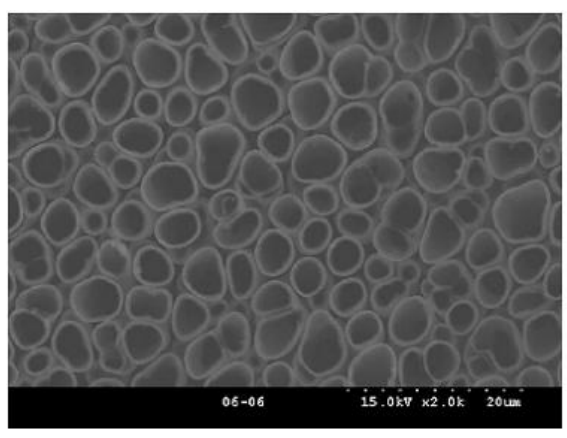

(b)

Fig. 9. Surface view of filter after process.

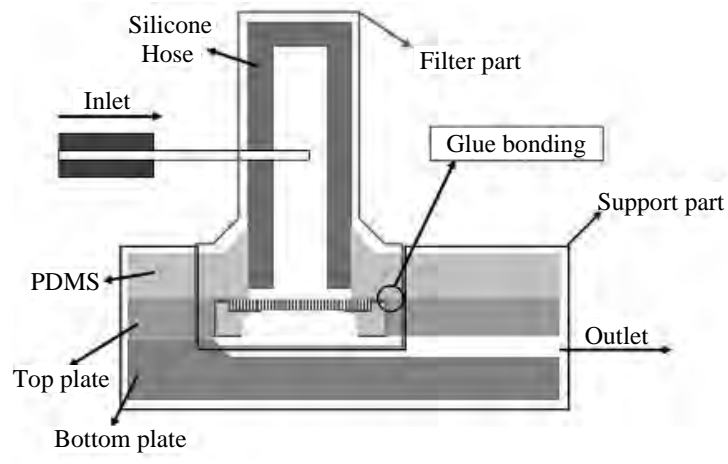

Fig. 10. Schematic of the fabricated filter combined with PDMS. 


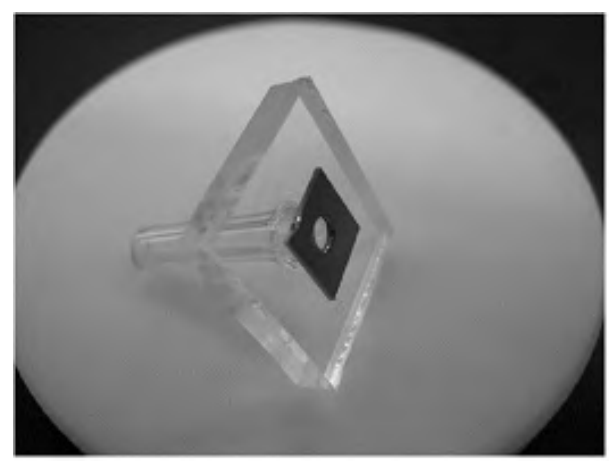

Fig. 11. Fabricated filter used for measurement purposes.

\subsection{Filtering blood using biofilter}

A syringe was filled with whole blood to operate the syringe pump. When the syringe pump was operated, specimens were filtered. A glass cover was placed within $1 \mathrm{~mm}$ from the bottom of the filter to collect specimens on the glass. This distance was maintained to prevent the membrane from being destroyed by pressure from the base plane when the filter membrane touched the glass cover. The filter had a pore diameter of $5 \mu \mathrm{m}$ or less for filtering red blood cells as well as white blood cells and screened blood platelets. ${ }^{(14)}$

Figure 12 shows microscopy images of blood, which does not coagulate in the presence of ethylenediaminetetraacetic acid (EDTA). Figure 12(a) shows an image taken prior to filtering the whole blood to confirm the presence of white and red blood cells and blood platelets. Figure 12(b) shows an image taken after filtering the whole blood. Platelets having a diameter of $2 \mu \mathrm{m}$ or less remained in the specimen after penetrating into the filter, while the white and red blood cells having diameters of more than $5 \mu \mathrm{m}$ were not filtered.

Blood without EDTA coagulated within several minutes because of the rapid coagulation of blood platelets, even upon sealing the glass cover. The blood was filtered and maintained at $15^{\circ} \mathrm{C}$ for approximately $3 \mathrm{~h}$ and examined under a microscope. Before filtering, as shown in Fig. 13(a), the blood was much more deformed than that shown in Fig. 12(a). The white blood cells maintained their shape, and the coagulation of the blood platelets occurred. Figure 13(b) shows the coagulated blood platelets in the filtered blood.

The blood was filtered immediately after extraction, so that all blood platelets penetrated into the filter in the filtering process, however, blood platelets coagulated when left to stand for $3 \mathrm{~h}$. 


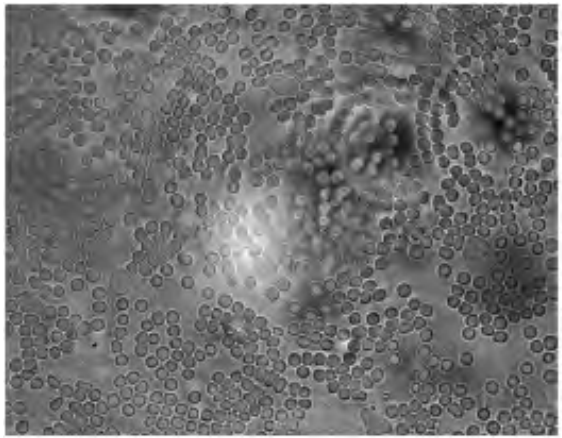

(a)

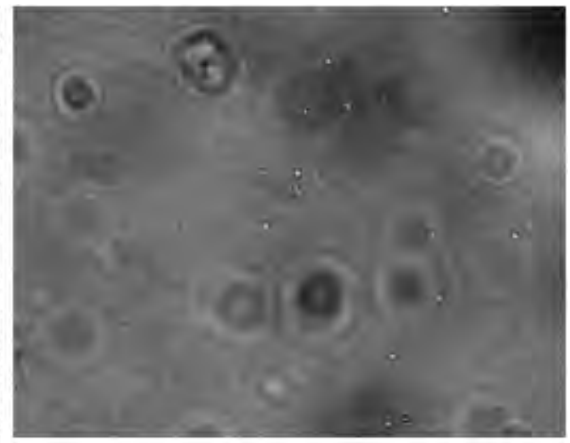

(b)

Fig. 12. Microscopy images of filtered blood: (a) primary blood and (b) filtered blood.

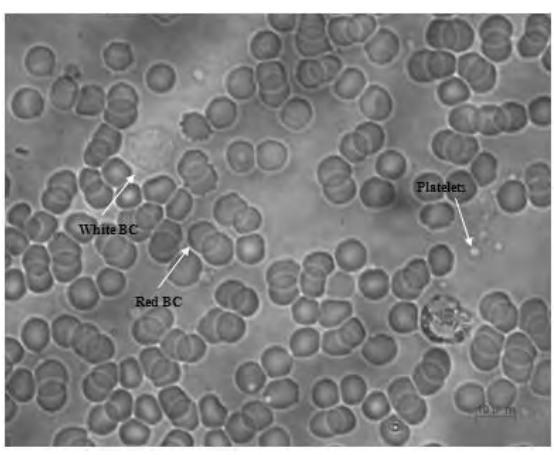

(a)

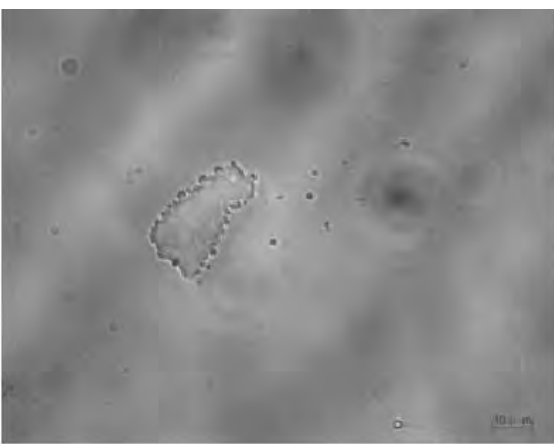

(b)

Fig. 13. Microscopy images of filtered blood without EDTA: (a) primary blood and (b) filtered blood.

\section{Conclusions}

In this paper, we fabricated a biofilter using a porous layer made by an anodic reaction of (110) silicon to increase the width of pores and decrease the width of the walls between pores. The filter used in a previous study had a porosity of $43 \%$ relative to that of the surface, while in this study, a filter with a maximum porosity of $73 \%$ was used. This excellent porosity resulted in not only large pores, but also thin walls between pores.

In the blood filtering examination, platelets having a diameter of $2 \mu \mathrm{m}$ or less remained in the specimens after penetrating into the filter, in contrast to white and red blood cells that have diameters of more than $5 \mu \mathrm{m}$, which did not remain. It is expected that the microfilter can be applied to lab-on-a-chip and micro-total analysis systems for the analysis of various materials. 


\section{Acknowledgements}

This research was supported by Kyungpook National University Research Fund, 2007.

\section{References}

1 K. Grigoras, A. Niskanen and S. Franssila: J. Micromech. Microeng. 11 (2001) 371.

2 R. T. Howe: J. Vac. Sci. Technol. B 6 (1988) 1809.

3 K. E. Bean: IEEE Trans. Electron Devices 25 (1978) 1185.

4 E. Bassous: IEEE Trans. Electron Devices 25 (1978) 1178.

5 A. Uhlir: Bell Syst. Tech. J. 35 (1956) 333.

6 L. Xiaowei, L. Xin, W. Wei, W. Xilian, C. Wei, L. Zhenmao and F. Maojun: Conf. Solid-State Circuit Technol. (1998) p. 891.

7 M. I. J. Beale, J. D. Benjamin, M. J. Uren, N. G. Chew and A. G. Cullis: J. Cryst. Growth 73 (1985) 622.

8 C. W. Wilmsen: Physics and Chemistry of III-V Compound Semiconductor Interfaces (Plenum Press, New York, 1985).

9 R. L. Smith and S. D. Collins: J. Appl. Phys. 71 (1992) R1.

10 X. G. Zhang: J. Electrochem. Soc. 151 (2004) C69.

11 V. V. Denyaka, I. G. Evseevb and V. M. Khvastunova: Braz. J. Phys. 33 (2003) 133.

12 M. J. Alguard, R. L. Swent and R. H. Pantell: IEEE Trans. Nucl. Sci. 26 (1979) 3865.

13 K. Grigoras, S. Franssila, T. Sikanen, T. Kotiaho and R. Kostiainen: Phys. Status Solidi A 202 (2005) 1624.

14 B. Alberts, D. Bray, A. Johnson, J. Lewis, M. Raff, K. Roberts and P. Walter: Essential Cell Biology: An Introduction to the Molecular Biology of the Cell (Garland, New York, 1997). 